\title{
USING HIGH RESOLUTION DATA TO INVESTIGATE THE VARIABILITY OF GG CARINAE SYSTEM
}

\author{
Maria Auxiliadora Machado, ${ }^{1}$ Francisco Xavier de Araújo, ${ }^{1}$ Dalton de Faria Lopes, ${ }^{1}$ and Claudio Bastos
}

Pereira $^{1}$

GG Carinae is a peculiar emission line object classified as a $\mathbf{B}[\mathrm{e}]$ star. Using high resolution data we confirm that GG Carinae is at same time a binary system and a variable star.

GG Carinae was classified by Lamers et al. (1998) as a B[e] star and also is pointed out as a binary system. So, any investigation to determine its parameters requires an detailed analysis in order to know and to separate the effects of each component. Gosset et al. (1984) used Fourrier Analysis in the data obtained from photoeletric photometry of both the standard UBV and Strongren system and calculated a period of $31^{D} .02$. According the authors there are two possible scenarios for GG Carinae: i) GG Carinae has an assymetrical atmosphere where the P Cygni profiles are formed and the brightness variation would be caused by extintion due it is moving inside a nebulae; ii) the companion of $G G$ Carinae must have a intense hydrogen-rich hot spot which orientation respect to observer would be responsible by the light variation. It is important to note that there is no conclusion about its binary nature as well as to the physical structure of his envelope. This unconclusive scenarium and the desire to take GG Carinae in account in our investigation about massive stars motivated us to analyse this star.

In this work we present recent optical high resolution observations taken over the lightcurve of GG Carinae obtained by Gosset et al. (1985). These spectra show new aspects about the binary nature of GG Car and in special about the intrisic variability of the main star wich has been predicted by Kruytbosch (1930).

The high resolution data were taken with the Fiber-fed Extended Range Optical Spectrograph (FEROS) that is a Echelle spectrograph with a spectral resolving power of $R=48000$ corresponding to 2.2 pixels of $15 \mu \mathrm{m}$ and with a wavelength coverage from $3600 \AA$ to $9200 \AA$.

The main aspects observed on the spectra of GG Carinae were:

1. The formation of a second absorption compo-

\footnotetext{
${ }^{1}$ Observatório Nacional/MCT, Rua General José Cristino 77, 20921-400,Rio de Janeiro, BRAZIL (dora@on.br).
}

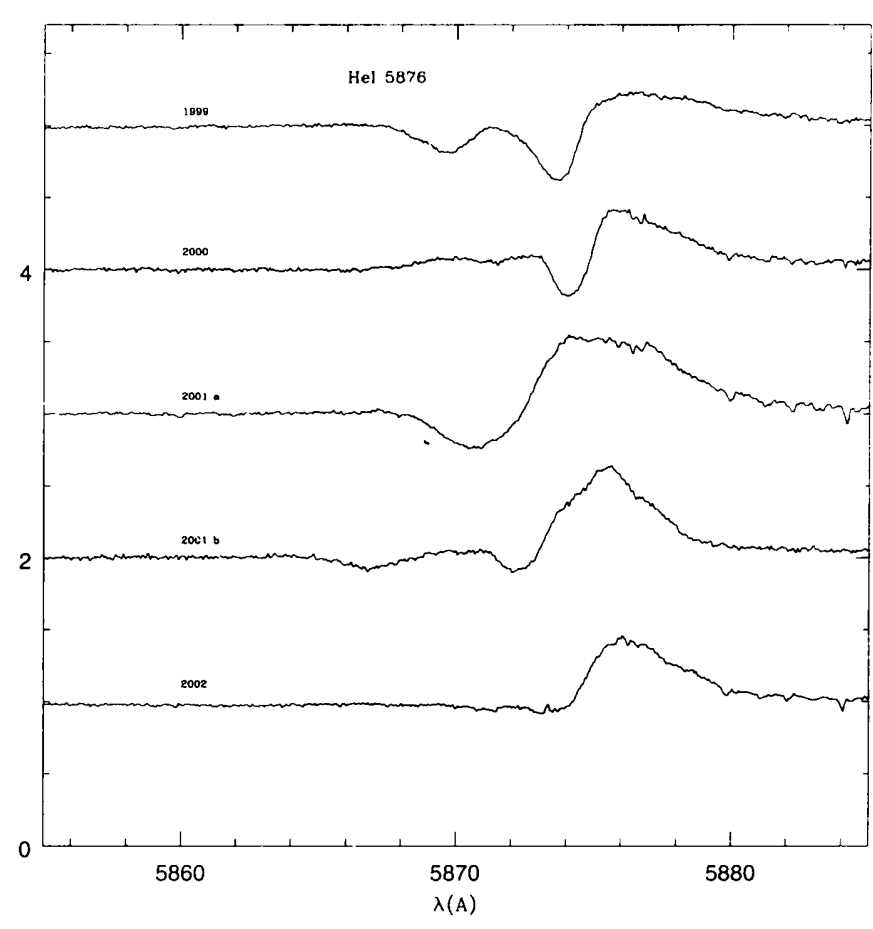

Fig. 1. Variations of HeI $5876 \AA$ over the time.

nent on Balmer and on some He I lines.

2. The variation on equivalent widths of Balmer. $\mathrm{He} \mathrm{I}$ and $\mathrm{Fe}$ II lines.

Analizing the phase variations for $H_{\delta}, H_{\gamma}$ and the He I lines, we can see that the second absorption component present in these lines are not in phase and cannot be associated to an ocultation. However. when we analyse this variability over the time, we see that the evolution of the shape of the profiles seems indicate a variation on density structure of the wind. Figure 1 show the variations of the HeI $\lambda 5876 \AA$ An the time. However the behavior of the stellar component of the resonance doublet of $\mathrm{Na} \mathrm{I}$ $\lambda \lambda$ 5890-5896 as well as the Fe I lines are also in phase, confirming the presence of a companion.

\section{REFERENCES}

Gosset,E., Hutsemékers,D., Surdej,J., \& Swings.J.P. 1985 A\&A, 153, 71

Kruythosch,W.E.,1930, Bull. Astron. Inst. Neth. 6, 11

Lamers,H.J.G.L.M., Zickgraf, F.-J., de Winter,D., Houziaux,L., \& Zorec,J. 1998 A\&A, 340,117 